\title{
La fotografía aplicada a la reproducción y restauración de obras de arte
}

\section{José Francisco Lorén González}

Arbor CLXIX, 667-668 (Julio-Agosto 2001), 591-198 pp.

En este artículo vamos a tratar, como el título nos da a entender, y brevemente, de las diversas técnicas de que nos podemos servir para documentar adecuadamente el proceso de restauración de una obra, sea pintura o escultura, y se haga este trabajo en el estudio o en el lugar en que se realiza la restauración, como puede ser un retablo o una pintura mural.

Empezaremos por referirnos al equipo a utilizar que en principio y por economía y versatilidad será de formato $35 \mathrm{~mm}$., que es el formato en el que casi todos tenemos cámara o equipo.

De momento vamos a dejar de lado la tecnología digital para ceñirnos al material de soporte químico, es decir, las películas normales y corrientes, tanto en negativo como en transparencia o diapositiva, aunque las técnicas a utilizar sean las mismas en los dos casos.

Empezando por el principio, lo ideal es contar con una cámara dotada de ópticas intercambiables, pues en función del tipo de fotografía que vayamos a hacer, necesitaremos desde objetivos gran angulares a teleobjetivos de una distancia focal moderada, con algún objetivo para macrofotografía.

Como objetivos grandes angulares podemos empezar con uno de distancia focal en torno a $28-35 \mathrm{~mm}$., aunque a veces, si tenemos que fotografiar un retablo entero o alguna zona amplia sobre el andamio el objetivo de $20 \mathrm{~mm}$., nos da unos resultados sensacionales.

Para hacer macrofotografía, cuando tengamos que hacer desperfectos o detalles que nos interesen los objetivos suelen oscilar entre unas 
distancias focales de 55/60 mm., y $105 \mathrm{~mm}$., pudiendo servirnos este primero como objetivo normal, pues sirven indistintamente para corta y larga distancia, y el de $105 \mathrm{~mm}$., nos puede servir, en cuanto a macrofotografía, para fotografiar zonas pequeñas sin acercarnos en exceso al objeto, y también para fotografiar motivos que estén a una distancia excesiva para utilizar un objetivo de distancia focal inferior.

Objetivos de focales superiores a éstas, como pueden ser 135 ó $200 \mathrm{~mm}$., son de muy escasa utilización.

El siguiente elemento a incluir en el equipo debe ser un trípode, que no tiene que ser muy pesado, pero si nos interesa que alcance una altura totalmente desplegado de 1,70 metros aproximadamente. Este trípode debe complementarse con un cable sincro que no tiene que ser muy largo.

Nos puede ser de utilidad un fotómetro para medir la luz incidente, sobre todo si utilizamos flash electrónico, pues el fotómetro de la cámara mide la luz reflejada y en el caso de utilizar flash, éste no nos sirve.

Para fotografiar podemos utilizar tres tipos de fuente de luz; en primer lugar la luz natural, que puede ser la luz ambiente de una escultura en un parque, un retablo en el interior de una iglesia o la luz que entra por el balcón de nuestro estudio.. En estos dos últimos casos nos podemos encontrar con problemas de cantidad y calidad de luz, aunque se podrían solucionar con exposiciones largas o utilización de filtros compensadores.

El segundo tipo de iluminación puede ser mediante la utilización de lámparas de incandescencia, que pueden ir desde la simple bombilla de la lámpara hasta sofisticados equipos de cuarzo con ventilación, etc., pasando por las veteranas lámparas nitraphot, de 250 ó $500 \mathrm{~W}$.

La tercera fuente de luz, es la del flash electrónico, muy cómodo y sin problemas de equilibrio de color, pues se utilizan las películas tipo luz días normales, de 5.500 grados kelvin.

Esta película también será la que utilizaremos en el caso de que queramos hacer fotografías con luz ultravioleta, solo que en este caso, las exposiciones serán largas en función del tipo de lámparas que utilicemos y la distancia a la que esté la obra a fotografiar.

Así como la luz visible es una radiación electromagnética que se extiende en una serie de longitudes de onda entre 400 y $700 \mathrm{~nm}$., la luz ultravioleta se extiende entre unas longitudes de onda de $10 \mathrm{y}$ $400 \mathrm{~nm}$., según sean ultravioletas de onda larga, media y corta, pero en nuestro caso solo utilizaremos la ultravioleta de onda larga, con unas longitudes de onda entre 300 y $400 \mathrm{~nm}$., áproximadamente. 
Podemos utilizar dos tipos de lámparas para iluminar; o bien los tipos fluorescentes tipo TLD, que pueden tener una longitud de 100 cm., o bien lámparas tipo incandescente, como las OSRAM HWV de $160 \mathrm{~W}$, con la pega de que son lámparas diseñadas para trabajar en vertical, por lo que si las utilizamos horizontales, que será lo más frecuente, tenemos que tener cuidado de no moverlas lo mas mínimo, porque con las vibraciones se apagan, y hasta que no se enfrían no vuelven a funcionar.

Son más cómodos los tubos, porque además reparten mejor la luz y no irradian calor, además se puede uno fabricar un equipo artesano por poco dinero.

A la hora de fotografiar, la técnica es la misma que con luz visible, solo que con exposiciones más largas que habrá que establecer mediante pruebas, pues los fotómetros habituales no sirven en estos casos.

También debemos utilizar de la cámara un filtro tipo KODAK Wratten $2 \mathrm{~B}$, que es el equivalente a las gafas que se supone utilizamos para trabajar con la luz ultravioleta.

El revelado de la película será el habitual.

A la hora de fotografiar una obra, empezando por las de tipo plano, a saber, murales, cuadros, una obra sobre papel, etc., lo ideal es disponer de 2 ó 4 puntos de luz que se colocarán simetricamente respecto a la obra, y teóricamente formando un ángulo de 45 grados, aunque esto es muy elástico, pues en función de los problemas que pueda dar la obra, en función de brillos, textura, etc., puede llegar a colocarse muy rasante, casi paralela a ella.

Uno de los principales problemas con los que nos vamos a encontrar a la hora de fotografiar un cuadro, es la aparición de brillos que algunas veces nos ayudarán a identificar desperfectos de la obra, pero la mayoría de las veces nos crearán problemas serios. En este caso, una solución sencilla es poner las luces más tendidas, es decir, formando un ángulo mayor de 45 grados con el eje del objetivo y utilizar un objetivo de la distancia focal más larga que nos permita el equipo o el espacio disponible.

Si con esto no conseguimos eliminar o reducir de forma significativa los brillos, deberemos recurrir a técnicas más radicales, como la utilización de luz polarizada. La luz ordinaria, no polarizada vibra en todas las direcciones perpendiculares a la de su desplazamiento. Cuando la vibración se limita a un plano tenemos luz polarizada.

Esta técnica consiste en la utilización de filtros polarizadores tanto en la fuente de luz como en la cámara. 


\section{José Francisco Lorén González}

Primero colocaremos los filtros en la fuente de luz. Estos filtros se pueden comprar en rollos o cortados en medida de 50x52 aproximadamente, son de gelatina, y se deben colocar a una distancia prudencial de la fuente de luz para que no se quemen con el calor.

El filtro que pondremos en la cámara, es de cristal, y deberemos hacerlo girar hasta lograr la total eliminación de los brillos.

La utilización de estos filtros absorbe mucha cantidad de luz; aproximadamente $1^{1 / 3}$ de diafragma los de gelatina y de $1^{2 / 3}$ a 2 diafragmas los de cristal de la cámara.

Esta técnica tiene la ventaja de eliminar prácticamente al 100 por ciento los brillos, pero como contrapartida se nos presentan casos en que el contraste puede variar, aumentándolo apreciablemente en función del tipo de pigmento, técnicas, barnices, etc.

Otra técnica muy común en fotografía de restauración es la utilización de luz rasante que nos puede dar de un vistazo una idea bastante precisa del estado de la obra, sea lienzo, papel o mural.

Para conseguir esto, debemos situar las fuentes de luz en un solo lado y muy lateral. Cuando más lateral la coloquemos mayor será el efecto conseguido.

El problema que nos puede presentar esto es el de la medición de la luz, pues los fotómetros normales no nos la medirán con precisión.

En este caso debemos sustituir la pieza semiesférica que cubre la célula del fotómetro, por la plana que suelen acompañar el fotómetro.

Así se pueden conseguir resultados espectaculares.

Cuando tengamos que trabajar con obras tridimensionales, esculturas, retablos, etc., la técnica es distinta. Aquí el concepto cambia radicalmente. En función del tipo de luz que utilicemos, y cómo la utilicemos (a lo largo de este artículo, ya os habréis dado cuenta de la importancia de la luz en la fotografía), conseguiremos unos resultados totalmente distintos. Podemos resaltar o suavizar las texturas, formas y volúmenes, según utilicemos una luz directa, dura y dramática, o bien la suavicemos mediante paraguas o cajas de luz.

$\mathrm{Si}$ queremos fotografiar una escultura completa lo ideal es disponer de un ciclorama o fondo continuo. Para el fondo continuo podremos utilizar unos rollos de papel especialmente fabricados para estos menesteres, que vienen en rollos de longitud variable y en ancho de 220 $\mathrm{cm}$., están fabricados en un papel grueso que soporta aceptablemente el peso de las obras, siempre y cuando el suelo esté liso, pues si no se marcarán los desperfectos o marcas que tenga el pavimento, estando disponible en una amplia gama de colores a fin de poder escoger el que entone mejor con la obra a fotografiar. 
La fotografía aplicada a la reproducción y restauración..

Deberemos tener cuidado para pisar lo menos posible el papel, pues parece mentira como se quedan marcadas las huellas del calzado. Esto lo podemos evitar colocando unos papeles de periódico o de cualquier otro tipo sobre el rollo de fondos, para colocar la obra y medir la luz y los quitaremos a la hora de efectuar la fotografía.

Una vez colocada la obra, el siguiente paso es la iluminación.

Como hemos dicho antes, esta puede ser de varios tipos, pero en general no debe ser muy dura, pues nos daría unos contrastes muy altos y unas sombras nada transparentes.

En este caso utilizaremos los paraguas o cajas de luz. Los paraguas, como indica su nombre, es una adaptación de estos artilugios para su utilización en fotografía, pueden ser de un tejido traslúcido en el que la luz del flash atraviesa el tejido, suavizándose la luz notablemente, o bien estar revestidos interiormente de un material blanco, para luz muy difusa; plateado, para una ilưminación un poco más enérgica; o incluso en algunos casos dorados, pero esto es básicamente para retratos al dar una luz muy cálida. También existen los de tipo reversible, blanco por un lado y plateado por el otro. Yo particularmente utilizo los plateados, pues son los que considero mas adecuados para una utilización general.

Las cajas de luz son un poco mas sofisticadas que los paraguas y también más voluminosas. Pueden ser de luz directa o de luz rebotada. En el primer caso, la luz sólo está tamizada por una ligera pantalla de tejido traslúcido y el objeto a fotografiar recibe la luz directa a través de la pantalla. En el segundo caso, la luz se dirige al fondo de la caja, (que en ambos casos está revestida con material blanco o plateado) y este revestimiento rebota la luz haciéndolo atravesar la pantalla traslúcida como en el caso anterior, pero más suave.

Según los efectos que queramos conseguir, deberemos escoger unos $\mathrm{u}$ otros elementos y colocarlos de forma que resalten el efecto que queramos conseguir. Deberemos utilizar una luz principal, y luego luces de relleno para aclarar sombras o conseguir efectos particulares. También en determinados casos deberemos utilizar una luz para aclarar el fondo, o separar el objeto mediante un ligero contraluz.

Otro sistema para aclarar determinadas zonas que no tengan suficiente iluminación es utilizar pantallas reflectoras, que pueden ser desde planchas de porexpan a cartón pluma de la dimensión que necesitemos. Este método podemos utilizarlo cuando tengamos que fotografiar alguna obra al sol, y nos dé unas sombras muy marcadas. Es un método que se utiliza mucho en cine, y da muy buenos resultados. 


\section{José Francisco Lorén González}

Si utilizamos luz directa, aparte de ser muy dura y con un contraste muy elevado (luces y sombras muy marcadas), nos dará en el papel de fondo unas sombras muy poco estéticas, y cuanto más puntos de luz, mas sombras antiestéticas, por solo es recomendable en casos muy concretos y específicos.

Si es una obra clara, como mármol lo madera clara, podemos utilizar un fondo negro, con lo cual las sombras se perderían en él, aunque debemos tener en cuenta que el fondo negro absorbe mucha luz por lo que tendremos que compensar la medición del fotómetro. Esto también tendremos que hacerlo (la compensación en la medición del fotómetro) cuando el objeto a fotografiar sea muy oscuro, como un bronce, pues si no corremos el riesgo de que nos salga un manchón negro.

En el caso de fotografiar bronces, cada acabado es un mundo, pues no es lo mismo fotografiar un bronce negro con una pátina superbrillante, que una obra de Miró con un acabado verde mate claro. En el primer caso debemos realzar las formas a base de jugar con los brillos, con luz mas bien suave, para que no nos queden esos puntos brillantes que sobresalen tanto en la foto. Esto mismo podemos aplicarlo a otros materiales con acabados brillantes y oscuros, como madera, hierro, poliéster o fibra de vidrio.

En el segundo caso el problema es menor, casi inexistente, y podemos tratarlo como cualquier otro material.

Si una vez fotografiada la pieza queremos hacer una serie de detalles, el tratamiento es distinto, aquí si que podemos utilizar luces directas si lo que queremos es resaltar texturas, desperfectos o detalles específicos, pues con este tipo de luz, los arañazos, las pérdidas o daños de otro tipo se identifican con mucha facilidad.

Aquí debemos tener en cuenta la pérdida de profundidad del foco que se produce al hacer macrofotografías, y la pérdida de luz por la ampliación del tiraje del objetivo, pues los objetivos macro al enfocar un objeto muy próximo aumentan su longitud, lo que lleva implicada una pérdida de luz que hay que compensar mediante unas tablas que suministra el fabricante con las instrucciones. Esto, por supuesto, también es aplicable a cualquier motivo que fotografiemos, ya sea plano o tridimensional.

Ahora vamos a tratar el tema de las fotografías con luz ambiente, es decir, en museos, salas de exposiciones, o en cualquier otro lugar cerrado, en el que nosotros no controlamos la cantidad ni la calidad de la luz. Si las obras que tenemos que fotografiar son pequeñas, no hay problema pues utilizaríamos nuestro propio equipo de iluminación, pero si son grandes nos encontramos con el problema de la falta de 
potencia. Esto se solucionaría si pudiéramos dejar la sala a oscuras, en este caso lo que haríamos sería utilizar la técnica de los disparos múltiples de flash.

Esta técnica consiste en dejar el objetivo abierto en la posición B o T, y disparar varias veces los flashes, pues las descargas de flash son acumulativas y si el fotómetro nos indica con un disparo de flash un diafragma de 4, con dos disparos tendremos un diafragma de 5,6, y si es con tres disparos de 8. Esto lo podremos hacer siempre y cuando la oscuridad sea total, pues si no es así, y al tener el diafragma abierto durante cierto tiempo, las luces parásitas nos pueden influir en el resultado, tanto en dominantes de color, como en sobre-exposición.

Si se trata de una obra muy grande, como una gran escultura o un pequeño retablo de una iglesia, y tenemos la inmensa suerte de que las paredes o el techo son blancos, podemos utilizar la técnica de la luz rebotada, que consiste en dirigir el haz de luz a dichas paredes o al techo. Esto nos produce una iluminación muy suave, que en determinados casos resulta satisfactoria, esto, claro está, solo podremos hacerlo siempre y cuando las superficies sean blancas, pues si no se nos pueden presentar unas dominantes de color muy desagradables.

Cuando no tengamos mas remedio que utilizar la luz ambiente, deberemos recurrir a otros métodos en función del tipo de luz que exista en la sala, aunque lo habitual es que haya una mezcla de luces que resulta un pastiche, pues pueden coexistir, luz natural con proyectores halógenos y fluorescentes de diversos colores a la vez, y esto no es nada infrecuente. Si disponemos de un termocolorímetro el problema queda resuelto, pero esto no es habitual, es un aparato caro y poco frecuente.

En esencia, este aparato es un analizador de luz para la medición de tres colores, que indica los filtros necesarios para obtener un resultado correcto. Esto se consigue mediante tres fotocélulas de silicio con filtros para detección de luces azul, verde y rojo. Un microordenador establece la composición de la luz, y la traduce a valores de filtros fotográficos «LB» (equilibrio de luces) y «CC» (composición de color). Pero como hemos dicho antes es un aparato poco frecuente.

Si la luz que tenemos es la luz natural que entra por ventanas, no hay problema, pues aunque tengamos que utilizar exposiciones muy largas por la pequeña cantidad de luz disponible, los resultados serán correctos, siempre y cuando tengamos en cuenta la pérdida de sensibilidad de la película por la ley de reciprocidad. Si la luz es mucha, mejor que mejor, no hay problema. 
Si la iluminación es mediante lámparas halógenas o de incandescencia, deberemos utilizar película equilibrada para una temperatura de color de $3200^{\circ} \mathrm{K}$, pero en película de $35 \mathrm{~mm}$., esto solo es posible cuando utilizamos diapositiva, pues en negativo no hay de este tipo. En este caso deberemos filtrar la luz mediante un filtro de tipo 80 $B$ de Kodak o similar, tendremos que tener en cuenta que con este filtro deberemos abrir un diafragma mas por la pérdida de luz.

Con las luces fluorescentes, los resultados son muy inciertos, pues hay infinidad de tipos y colores, en general se utilizan filtros de color magenta, como un $30 \mathrm{M}$, que absorbe un $2 / 3$ de diafragma, pero ya hemos dicho que con resultados, en general, poco satisfactorios, y si ya pasamos a lo mas habitual, que es la mezcla de varias de estas fuentes de luz, lo mejor es hacer antes unas pruebas. 